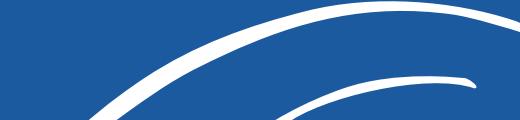 water and landscape AGUA Y TERRITORIO
}

\section{Primer ciclo de planificación hidrológica en España en aplicación de la Directiva Marco del Agua}

\author{
Observatorio de las Políticas del Agua (OPPA) \\ de la Fundación Nueva Cultura del Agua (FNCA)
}

ASPECTOS JURÍDICOS, DE PROCEDIMIENTO

Y DE PARTICIPACIÓN PÚBLICA

\section{Aprobación de los planes hidrológicos}

El primer ciclo de planificación en España se ha caracterizado por la acumulación de considerables retrasos que han limitado la efectividad práctica del mismo. Estos retrasos han afectado a todas sus fases. Por ejemplo, la Junta de Andalucía creó la Comisión de Autoridades Competentes para las tres demarcaciones hidrográficas intracomunitarias andaluzas con ocho años de retraso en 2012, dos años después de que el Tribunal de Justicia condenara a España por incumplimiento de dicha obligación. La aprobación de los planes hidrológicos de las demarcaciones españolas se ha postergado hasta reducir su periodo de vigencia a un escaso testimonio. La falta de entrada en vigor de los planes hidrológicos o su inaplicación cuando están aprobados (ej. por falta de fondos) está lastrando de manera determinante el cumplimiento de las obligaciones de protección y uso sostenible de la política de aguas. Destaca el caso de las demarcaciones de Canarias, que no tienen aún sus correspondientes planes aprobados.

El retraso generalizado en los calendarios de implementación de la DMA tiene también importantes consecuencias para el segundo ciclo de planificación. Así, los programas de medidas no han tenido el tiempo suficiente para ser aplicados (caso de los PH del Ebro, Júcar, Segura o Tajo), por lo que tampoco van a poder ser evaluados, lo que a su vez impide la adecuada revisión de cara al segundo Plan ya en marcha. De hecho, en su mayoría, los documentos disponibles del nuevo ciclo de planificación 2015-2021 (básicamente los Esquemas de Temas Importantes, ETI) son casi idénticos a los anteriores (2014-2015), lo que supone dar por perdido el primer ciclo de planificación y desaprovechar el aprendizaje que posibilita el enfoque de ciclo de planificación que plantea la DMA.
Por otra parte, en el caso de algunos planes hidrológicos se han incumplido los procedimientos establecidos por la DMA para su elaboración. Un caso emblemático es el de la demarcación de Baleares. En febrero de 2011 fue aprobado con carácter inicial el Plan Hidrológico de Baleares, quedando solo pendiente de su aprobación por el Consejo de Ministros. Sin embargo, tras el cambio de gobierno balear en mayo de 2011, se solicitó la devolución del Plan y en 2013 se aprobó definitivamente un nuevo texto claramente desregulador, que eliminó de la Normativa una parte sustancial de las medidas previamente incluidas. Las alegaciones aceptadas en el plan de 2011 y en parte incorporadas a la normativa de 2011, se han ignorado en la nueva Normativa, de la que se elimina el $30 \%$ de su articulado, sin dar respuesta motivada de las causas de su supresión. El nuevo texto del Plan no fue sometido a exposición pública y en ningún momento se aporta justificación técnica alguna de los cambios realizados al texto aprobado en 2013. Todo ello crea una gran inseguridad jurídica y representa un grave fraude administrativo que incumple el artículo 14 de la Directiva 2000/60/CE y el artículo 9 de la Directiva 2001/42/ CE. Todo ello ha llevado a que con fecha 17 de octubre de 2014 la Comisión Europea haya comunicado la admisión a trámite de las denuncias presentadas por diversos grupos y colectivos (Profesores universitarios y otros particulares - REF. CHAP (2014)1100; GOB y otros grupos ecologistas y colegios profesionales- REF. CHAP(2014)112 y Ecologistas en Acción- Ref.CHAP(2014)01217) por incumplimiento de la normativa comunitaria del Plan Hidrológico de Baleares.

También en el caso del Guadalquivir se producen profundas modificaciones en los contenidos del Plan definitivamente aprobado respecto al borrador sometido a información pública entre diciembre de 2010 y junio de 2011. Estos cambios se refieren especialmente al Estuario del Guadalquivir, sin mediar información y proceso de participación pública sobre estos nuevos contenidos ni explicar adecuadamente el motivo de las sustanciales modificaciones introducidas. 
El incumplimiento de la DMA ha dado lugar a acciones jurídicas en otros casos como el Plan Hidrológico del Júcar, donde el Tribunal Supremo (TS) ha admitido a trámite un recurso interpuesto por Ecologistas en Acción contra el Real Decreto 595/2014, por el que se aprobó el Plan Hidrológico de esta demarcación. Entre los incumplimientos señalados en este recurso figuran los grandes retrasos de calendario (pese a que fue declarada Cuenca Piloto para una aplicación temprana de la Directiva), la demora no justificada para alcanzar los objetivos ambientales y el no establecimiento de caudales ecológicos en algunas masas de agua, entre otros aspectos.

\section{Participación pública}

En algunas demarcaciones se han llevado a cabo procesos de participación pública ambiciosos y en línea con el espíritu de la DMA, particularmente en el caso de Cuencas Internas de Cataluña (la cuenca con mayor número de reuniones participativas), el Ebro (la cuenca con mayor número de participantes) y el proceso de participación pública en Baleares hasta el año 2011. Sin embargo, en muchas demarcaciones tales procesos se han abordado con un perfil muy bajo, de forma rutinaria, con escaso interés y sin identificar a los actores relevantes en el ámbito social.

La principal carencia de la gran mayoría de los procesos de participación pública puestos en marcha ha sido su falta de efectividad para influir realmente ante las cuestiones de calado, como la consecución de los objetivos ambientales, los programas de medidas y los costes. Cabe señalar como una de las excepciones más notables el caso de la primera versión del Plan Hidrológico de Baleares, con aprobación inicial en 2011, donde se incorporaron más del $80 \%$ de las alegaciones, integrándose parcialmente en la Normativa, desestimándose el resto con respuesta motivada. Sin embargo, el denominador común es que el proceso formal de participación pública apenas haya sido tenido en cuenta en los contenidos de los planes. El resultado es que la participación pública en el primer ciclo de planificación mayoritariamente no se ha aprovechado para mejorar la calidad de los planes, defraudando las expectativas ciudadanas y de muchos actores participantes. Por el contrario, los actores tradicionales del sector agrícola, industrial o energético, han dispuesto en muchos casos de vías paralelas de negociación con la Administración.

El incumplimiento de los procedimientos establecidos por la DMA para la planificación hidrológica y la ausencia de participación pública en los temas clave se ejemplifica de forma paradigmática en el caso de la demarcación del Tajo. El primer borrador del Plan Hidrológico se puso a disposición del público el 21 de octubre de 2011 pero se eliminó de la web del organismo de cuenca un día después. El nuevo borrador sale a consulta pública en marzo de 2013 con importantes cambios respecto al borrador anterior, como sustituir la propuesta previa de caudales ecológicos por unos caudales que denomina como "mínimos" y renunciar a cuestionar la viabilidad del trasvase Tajo-Segura. Estos cambios están relacionados con el proceso de negociación con los beneficiarios del trasvase Tajo-Segura al margen del proceso formal de planificación, el cual culmina con la incorporación de los contenidos del Memorándum del Tajo como enmiendas a la Ley de Impacto Ambiental de diciembre de 2013 (Ley 21/2013). Este Memorándum y las modificaciones normativas en tramitación se han mantenido en secreto y no se han difundido públicamente, quedando al margen de los órganos y autoridades competentes de la Demarcación Hidrográfica del Tajo así como del proceso de consulta y participación pública. Las modificaciones introducidas afectan de forma sustancial al cumplimiento de los objetivos ambientales en la demarcación del Tajo y suponen un flagrante incumplimiento de la DMA, particularmente de sus artículos 1, 4, $7.3,9,11,13.5,14$ y 15 .

\section{Coordinación interadministrativa}

En la gran mayoría de demarcaciones intercomunitarias en España, se constata la carencia, en muchas ocasiones, de elementales procesos de coordinación interadministrativa. Las Confederaciones Hidrográficas han sido las responsables de continuar ejecutando las actuaciones, mientras que los Comités de Autoridades Competentes (CAC) se han limitado a aprobar los documentos propuestos por los organismos de cuenca, sin una real participación en su elaboración y posterior seguimiento. Esta situación se repite en la gran mayoría de demarcaciones. En el caso del Ebro, el CAC no ha evitado la importante descoordinación entre el organismo de cuenca y las comunidades autónomas implicadas, en particular la Generalitat de Cataluña, tal y como evidencian las Actas de los Consejos del Agua de esta Demarcación. Algo similar ocurre en el caso del Guadalquivir, donde en el Consejo del Agua la Junta de Andalucía votó en contra del Plan Hidrológico de la Demarcación.

Además, no existe información disponible al público de las reuniones del Comité de Autoridades Competentes (orden del día de las reuniones, actas de las mismas).

En el caso de las cuencas intracomunitarias, la situación de falta de coordinación administrativa ha sido también generalizada, independientemente del órgano responsable de la gestión del agua. En términos generales se puede afirmar que los planes hidrológicos incorporan los programas sectoriales de las otras administraciones sin un análisis de su compatibilidad con los objetivos ambientales del plan.

La principal descoordinación tiene lugar entre las distintas políticas que se aplican en las Demarcaciones. Por ejemplo, existe una clara desconexión entre la gestión del agua y la ordenación urbanística y territorial, con el consiguiente aumento de la vulnerabilidad frente a la inundación. Esta desconexión se evidencia en demarcaciones como la del Guadalquivir, donde no existe una coordinación entre los contenidos de los documentos de ordenación territorial y el plan hidrológico. Entre otros ejemplos, el Plan Hidrológico no incorpora adecuadamente en su normativa las determinaciones del Plan de Ordenación del Territorio de Andalucía (POTA) y planes subregionales respecto a la red de drenaje, especialmente el artículo 90 del POTA. 
En la Demarcación del Segura se presentan inconsistencias incluso entre los propios documentos de planificación hidrológica. Por poner un ejemplo, en la demarcación del Segura los déficit hídricos que resultan de la comparación entre recursos y demandas se basan en valores medios interanuales desconectados de las medidas de restricción del suministro contempladas en el propio proceso de planificación, como las establecidas en el Plan Especial frente a la Sequía (PES), medidas cuya consideración reduciría la cuantificación del déficit medio interanual.

En relación con la parte portuguesa de las cuencas internacionales (Miño, Duero, Tajo y Guadiana), cabe destacar el Decreto-Ley sobre el régimen económico financiero, que establece las tasas a pagar por el uso privativo del agua por parte de los "grandes usuarios" (cuyas actividades causan mayores impactos en el medio hídrico) y las tarifas a pagar por el público en general (servicios de abastecimiento y saneamiento), decreto en línea con la DMA. Sin embargo, por exigencias de la "Troika" se eliminaron 22 organismos públicos, incluyendo los cinco organismos de cuenca (Administração da Região Hidrográfica) que se habían creado en 2007 para adaptar la administración del agua portuguesa a los requisitos de la DMA.

Esto ha interrumpido el esfuerzo de cooperación con los municipios que se inició para solucionar los problemas de aplicación de la legislación del agua, trabajo que no es probable que se pueda realizar desde un ente centralizador. En lo que se refiere a la cooperación hispano-lusa, es urgente mejorar la misma y que, idealmente, el siguiente ciclo (el ciclo 2021-2027, ya que los borradores de los planes hidrológicos para 2015 serán previsiblemente publicados en España en diciembre de 2014) de planificación se realice de modo conjunto.

\section{EVALUACIÓN DEL ESTADO DE LA CUENCA Y ANÁLISIS DE PRESIONES}

En muchas demarcaciones, como la del Segura y otras, siguen existiendo claras carencias y enormes dificultades para que los ciudadanos puedan acceder a la información relativa al uso y control del Dominio Público Hidráulico y a los usos responsables de las mayores presiones sobre el estado de las masas de agua, particularmente el regadío. La situación en la mayoría de las demarcaciones es la existencia de importantes trabas para acceder a la información sobre concesiones y captaciones de aguas superficiales y subterráneas, lo que dificulta enormemente que los ciudadanos puedan verificar la legalidad o no de una determinada extracción de agua o perímetro de riego.

Por otra parte, no está claro qué criterios se han utilizado para definir las presiones significativas en los diferentes planes, sorprendiendo que, por ejemplo, en el Plan Hidrológico del Ebro se considere que 225 presas, 850 azudes en cauce y 10.000 pequeñas balsas de regulación no representan una presión significativa. Igualmente en el Plan del Ebro no aparece ninguna referencia al lindano, considerando nula la presión por vertido de sustancias peligrosas, pese a los numerosos análisis que demuestran la existencia de esta sustancia en distintos puntos de la Demarcación, como los residuos de los vertederos de Sardas y
Bailín, los sedimentos contaminados en el embalse de Sabiñánigo y el río Gállego, que están afectando incluso al abastecimiento de agua de boca en las poblaciones afectadas.

Además, existe un inadecuado tratamiento y ausencia de interrelación entre los impactos detectados y las presiones de origen antrópico responsables de tales impactos. Por ejemplo, las presiones más generalizadas en la Unión Europea son las alteraciones hidromorfológicas. A su vez, en España existe una tendencia desde principios del siglo XX a identificar gestión de aguas con programación de obras hidráulicas públicas. Pese a ello, estas presiones y sus impactos no están adecuadamente identificados y analizados en la mayoría de los planes de las demarcaciones españolas. Igualmente, en la mayoría de planes hidrológicos (véase el caso del Segura, del Guadalquivir o del Guadiana) las presiones de origen agrícola aparecen pobremente diagnosticadas e insuficientemente conectadas con los impactos - cuantitativos y cualitativos - que generan sobre las masas de agua.

En relación con la necesidad de un análisis estratégico de los efectos de los trasvases en las cuencas cedentes, incluyendo los efectos de los caudales reducidos o modificados y otros aspectos del estado ecológico de todas las masas de aguas y los lugares Natura 2000, dicho análisis es inexistente, incluyendo el caso más emblemático: el trasvase Tajo-Segura. Este trasvase se ha convertido en una condición sine qua non, que condiciona la planificación y gestión de toda la demarcación del Tajo, afectando sobremanera el estado de muchos tramos de la demarcación, por lo que no se entiende que no se identifique como tema importante y que no aparezca en el Análisis de Presiones e Impactos.

\section{Aplicación del aRT. 9 Y ReCUPERACIÓN DE COSTES}

Se vienen desarrollando estudios económicos sistemáticamente sesgados y erróneos para justificar los proyectos en tramitación, con el fin de ocultar la enorme subvención que disfruta el agua de riego con aguas superficiales. En el caso de la cuenca del Ebro, con un pago medio del agua para regadío de 0,011 $€$ / $\mathrm{m}^{3}$, se pretende estar consiguiendo un nivel de recuperación de costes del 80\%; mientras que el coste medio calculado de forma rigurosa en los grandes proyectos de regulación y nuevos regadíos en la Cuenca del Ebro durante las dos últimas décadas se mueve entre 0,15 y $0,25 € / \mathrm{m}^{3}$.

Los resultados en regadíos con aguas superficiales que se presentan oficialmente con niveles de recuperación de costes por encima incluso del $80 \%$, son falsos. Los estudios al respecto más fiables sitúan la recuperación de costes en las aguas superficiales de riego en menos del 10\%, una vez se corrigen una serie de errores y trampas contables como las siguientes: a) inadecuados plazos de amortización; b) los fondos europeos son administrados como subvenciones a fondo perdido; c) no se asumen costes adecuados de mantenimiento y reposición de infraestructuras y d) se aplica un descuento correspondiente a la laminación de avenidas como forma de encubrir una subvención pública arbitraria, dado que dicho descuento no se corresponde con la capacidad de re- 
gulación reservada efectivamente a tal objetivo de laminación de avenidas.

Esta tasa de descuento en presas a cuenta de la laminación de avenidas, fijado de forma arbitraria, es un denominador común, con valores que por ejemplo en el caso del Guadiana representan un $50 \%$ de los costes de las grandes infraestructuras.

En relación con el análisis Coste-Efectividad y su aplicación en los programas de medidas, dicho análisis ha sido ignorado en los proyectos que se impulsan, dado que sistemáticamente se restringen las pretendidas alternativas efectivas a un abanico sesgado que ignora opciones que, siendo efectivas, son más económicas, de forma que la pretendida elección queda viciada por el propio abanico de opciones presentadas. Por ejemplo, en el Plan de Saneamiento y Depuración de Aragón se han sobredimensionado las depuradoras y se ha vetado considerar siquiera las opciones de depuración extensiva en núcleos urbanos de menos de 3000 habitantes. Ello ha llevado a encarecer los servicios de saneamiento, hasta un $1000 \%$ sobre el coste que se derivaría de haber aplicado el principio coste-efectividad. Otro caso ilustrativo, con financiación europea, es el abastecimiento de aguas a Zaragoza y su entorno, con ramales de hasta $50 \mathrm{~km}$ de longitud y desniveles a vencer de más de trescientos metros para llevar caudales desde el embalse de Yesa (que se pretende recrecer), ignorando la disponibilidad de caudales de calidad que podrían servirse por gravedad, de forma efectiva y mucho más económica.

En relación con los costes ambientales y del recurso, como se ejemplifica en el Ebro, Guadiana, Segura y generalidad de demarcaciones, no se han considerado costes ambientales en la tarifación de los servicios de riego, ni se han estimado los costes inducidos por la contaminación difusa, tanto en nitratos y pesticidas, como en sales. En cuanto a la medición de los volúmenes usados, si bien es mayor en los nuevos regadíos o en regadíos modernizados, las tarifas y cánones siguen aplicándose fundamentalmente por superficie regada.

La insuficiente e inadecuada repercusión de los costes provoca elevados costes sociales. No sólo por la traslación de las cargas de negocios privados al conjunto de los ciudadanos, sino también porque se excluyen alternativas más costo-eficientes. Es el caso, por ejemplo, de las desaladoras del Vinalopó (y otras cuencas mediterráneas), financiadas con fondos europeos y en buena parte ociosas porque, en ausencia de una política de recuperación de costes (incluidos los ambientales) general, no resultan una alternativa atractiva para unos usuarios que acceden a otras fuentes fuertemente subvencionadas.

\section{EstAdo ECOLÓgICO Y OBJETIVOS AMBIENTALES}

Existe un gran número de masas carentes de definición de estado ecológico, particularmente en las demarcaciones del Ebro (53\% de las masas superficiales no evaluadas, incluyendo la mayoría de masas de un espacio protegido tan emblemático como el Delta del Ebro), Guadalete-Barbate, Baleares, Galicia-Costa, Júcar y en la del Tinto, Odiel y Piedras, caso este último donde las masas cuyo riesgo de incumplimiento no ha sido evaluado asciende al $73 \%$.

Existen lagunas y carencias en el establecimiento de las condiciones de referencia, por ejemplo en el caso del Guadalquivir para algunas masas tipo río, en el Plan del Guadiana para casi todos los tipos de lagunas o en el Ebro, donde no hay condiciones de referencia para aguas de transición, aguas costeras y buena parte de los lagos.

Por otra parte, los planes no incluyen algunos indicadores clave de calidad requeridos por la DMA, tales como fauna ictiológica o parámetros hidromorfológicos (por ejemplo de alteración hidrológica). En la mayoría de demarcaciones no se aplican indicadores de peces (caso del Duero, Guadalquivir, Tajo, Guadiana, Ebro, Segura y otras); tampoco hidromorfológicos, salvo algún caso como el Duero, donde se aplican indicadores hidromorfológicos más rigurosos que en otras demarcaciones y donde los objetivos están vinculados a indicadores con valores concretos.

En buena parte de las demarcaciones se han aplicado prórrogas generalizadas para alcanzar el buen estado de las masas de agua, como ocurre en el Duero, el Ebro, el Guadiana (en la cuenca alta del Guadiana se han aplicado prórrogas al $100 \%$ de las masas subterráneas), la demarcación del Tinto, Odiel y Piedras, donde el 39\% de las masas de agua incumplirán el buen estado en 2015, el Júcar, donde dicho porcentaje sube al 53,5\% o el Segura, donde el $53 \%$ de las masas superficiales y el $73 \%$ de las masas subterráneas no alcanzarán el buen estado en 2015. Además, no se establecen en general objetivos intermedios ni análisis de eficacia de las medidas, lo que genera dudas acerca del logro de los objetivos en 2027.

\section{Caudales ecológicos}

Las propuestas de caudales siguen el esquema establecido desde la Dirección General del Agua orientado a adaptarlas a las necesidades de los usuarios, no existiendo una justificación clara de cómo se derivan dichas propuestas a partir de los trabajos técnicos disponibles. Por ejemplo, las diferencias entre demarcaciones en el caudal propuesto relativo (QPR) parecen contener una arbitrariedad elevada y estar influenciadas por las demandas hídricas para riego y otros usos.

En muchas demarcaciones (como la del Tinto, Odiel y Piedras, Guadiana, Segura, Tajo), no existe vinculación entre los caudales ambientales y el estado de las masas de agua o son claramente incoherentes, incluso cuando existen datos suficientes acerca de su estado: en el caso del Tajo, el borrador de Plan hidrológico sometido a consulta pública en 2013 modificó los caudales en el eje central del Tajo previamente propuestos, sustituyéndolos por unos caudales "mínimos" constantes a lo largo del año (una figura que no existe en la Instrucción de Planificación Hidrológica), muy inferiores al régimen de caudales ecológicos establecidos en el ETI y en el primer borrador del Plan.

En el caso del Segura, sólo en el $24 \%$ de las masas de agua tipo río se establece algún valor de caudal ambiental. En el caso del Júcar, solamente se definen caudales ecológicos para el $25 \%$ 
de los ríos en el horizonte de 2021. En el caso del Guadalquivir, los caudales ambientales mínimos se reducen a la mitad de los fijados por el Plan Hidrológico vigente (1998). En esta demarcación, en muchas ocasiones el régimen propuesto produce un hábitat insuficiente para el desarrollo de la vida piscícola, al proporcionar valores inferiores al $50 \%$ del hábitat que se puede alcanzar en los tramos.

Por otra parte, en la mayoría de demarcaciones la concertación de caudales ha sido insuficiente 0 inexistente, como en el caso del Duero, Tajo, Segura o Guadalquivir.

\section{Zonas protegidas}

En relación con el cumplimiento del artículo 4.1.c de la DMA respecto a las Zonas Protegidas bajo la Directiva de Hábitats, hay que decir que, en las demarcaciones de Galicia Costa y del MiñoSil, se han incluido objetivos específicos para las masas de agua en zonas protegidas, pero en la mayoría de las demarcaciones (como Cuencas Mediterráneas Andaluzas, Tinto, Odiel y Piedras, Guadalquivir, Segura, Tajo o Guadiana) no aparecen objetivos de conservación de las masas de los lugares Natura 2000.

Por ejemplo, en el caso del Tajo, no se analizan adecuadamente los requerimientos de las masas de aguas y especies protegidas, ni de los espacios incluidos en la Red Natura 2000. En estos momentos la Comisión tiene en sus manos una Queja interpuesta por los colectivos ciudadanos del Tajo [CHAP(2013)03137], referente a los impactos concretos que la gestión del río produce en los distintos espacios naturales de la Red Natura 2000. En el PH del Tajo, los apartados 2 y 3 del artículo 12 de la Normativa, acerca de nuevas infraestructuras en zonas Red Natura vulneran, como mínimo, los artículos 4.1, 4.7, 4.8 y 4.9, 11 y 14 de la Directiva Marco del Agua en lo relativo a las condiciones para las nuevas modificaciones 0 alteraciones de las masas de agua.

En la demarcación del Segura, un 68\% de las masas de agua tipo río están incluidas en la Red Natura 2000 y otros espacios naturales, pese a lo cual el Plan no realiza diagnóstico alguno de la relación entre las masas de agua y tales espacios ni establece medidas específicas para garantizar los niveles de caudales y calidad del agua requeridos en tales espacios.

En el caso de Baleares se da una situación paradójica. Por un lado la Memoria sí establece la relación entre las zonas protegidas y las masas de agua de la demarcación, así como los requerimientos hídricos cuantitativos necesarios para su gestión. Sin embargo, la Normativa, aprobada en 2013 tras el cambio de gobierno balear, contradice estas determinaciones, pues permite un incremento de extracciones en las masas de agua subterránea, sin tener en cuenta las limitaciones previamente establecidas en las zonas protegidas.

La situación de espacios protegidos tan relevantes como Doñana o l'Albufera de Valencia igualmente evidencian la desconexión entre los planes hidrológicos y las necesidades hídricas de los espacios protegidos de la Red Natura 2000.

\section{Excepciones}

En relación con las exenciones bajo los artículos 4.4 y 4.5 , en la generalidad de planes hidrológicos la prórroga de los plazos no se justifica en base a costes desproporcionados sino con una alusión genérica a la falta de disponibilidad presupuestaria, como ocurre en el caso del Duero, en la demarcación del Tinto, Odiel y Piedras, Cuencas Mediterráneas Andaluzas, el Guadiana, el Segura y el Guadalquivir, caso este último donde además la inversión en las medidas se reduce en un $53 \%$ respecto al borrador del Plan.

Con respecto a las exenciones por nuevo deterioro de las aguas si la actuación realizada cumple determinadas condiciones (art. 4.7), en España se omite su carácter excepcional y se concibe como una mera actuación que está sometida a condiciones.

La demarcación del Cantábrico Occidental destaca por haber sido la única en que además de identificar nuevas modificaciones 0 alteraciones, se han consignado los motivos que los justifican de forma expresa. Por el contrario, la mayoría de los planes hidrológicos españoles han aplicado esta exención omitiendo la obligación de consignar y explicar los motivos en cada uno de los casos. El caso Balear es llamativo, pues ni siquiera se menciona esta exención en el plan.

Por otra parte, el incumplimiento del plazo en el que debían aprobarse los planes (22.12.2009) también ha supuesto un incumplimiento en las obligaciones de exigir la prohibición del deterioro del estado de las masas de agua, ya que los planes no retrotraen la aplicación de las exenciones por nuevo deterioro de las aguas al menos a dicha fecha.

\section{Programas de medidas y programas de Seguimiento}

\section{Programas de medidas}

La mayoría de los Programas de Medidas han incumplido sistemáticamente los plazos establecidos por la Directiva. En muchas demarcaciones, con planes aprobados en 2013 o 2014, su aplicación será virtual y retórica en todo lo que no se corresponda con proyectos ya en marcha ajenos a la Directiva, dado que el Plan ha sido aprobado cuando su periodo de vigencia y el plazo establecido para alcanzar el Buen Estado, están prácticamente agotados.

En general los programas de medidas están condicionados por la herencia de políticas anteriores, son confusos en cuanto a sus objetivos predominantes, se han establecido sin la exigida evaluación coste-eficacia y carecen de un calendario suficientemente clarificado de ejecución y financiación.

Por otra parte, la vinculación de las medidas con los objetivos ambientales es muy vaga y genérica, no estableciéndose a escala de masa de agua y no respondiendo a los problemas específicos de las mismas en cuanto a su estado ecológico.

Una de las cuestiones básicas es el tipo de priorización de medidas que se establecen y en qué grado responden a medidas 
básicas bajo la DMA o se trata de proyectos de infraestructuras y otras actuaciones complementarias cuya finalidad no es necesariamente la de alcanzar los objetivos de la DMA. En este sentido, en muchas demarcaciones las medidas se aplican o no independientemente de su inclusión en el mismo, de la fase o estado de aprobación del Plan, y de su eficacia para conseguir los objetivos del Plan.

Además, los planes hidrológicos no suelen evaluar el impacto de las distintas medidas, ya sea positivo o negativo, respecto a los objetivos de alcanzar el buen estado. Entre otros muchos ejemplos, en la Demarcación del Tinto, Odiel y Piedras se prevé la construcción de los embalses de Alcolea, la Coronada y Pedro Arco, a ubicar en masas en estado peor que bueno, sin evaluar el impacto que puedan tener en la no consecución del buen estado ecológico. En la demarcación del Miño-Sil las medidas programadas inciden en la vieja política estructuralista en ámbitos como el abastecimiento, donde no se menciona la gestión de la demanda, 0 en la prevención de inundaciones, donde se proponen más presas de laminación sin analizar otras alternativas potencialmente más costo-eficaces como la gestión del territorio fluvial. En el caso de Galicia Costa el programa de medidas es más bien un catálogo de obras hidráulicas, sin relación concreta con los objetivos del Plan ni priorización.

En el caso del Segura, pese a que es una de las cuencas más reguladas del mundo, el Plan sigue incluyendo la construcción de nuevas obras de regulación, como el Plan de defensa contra avenidas del Alto Guadalentín o el Plan de actuaciones para el incremento de la laminación de avenidas y regulación en el río Mundo. Este patrón es general para la gran mayoría de demarcaciones. La cuenca del Ebro es un ejemplo emblemático de disociación entre estado de las masas, objetivos ambientales y medidas previstas: se incluye un amplio catálogo de obras hidráulicas así como ampliaciones de regadío, que de facto empeorarán el estado ecológico de las masas de agua, mientras que para algunas masas de agua en mal estado no se establece ninguna medida.

En términos presupuestarios, la inversión asignada a medidas para alcanzar el buen estado ecológico es mucho menor que las dedicadas a la satisfacción de las demandas. En el caso de la demarcación del Tinto, Odiel y río Piedras, sólo el 11\% del presupuesto del Programa de Medidas se destina al cumplimiento de los objetivos ambientales. En la demarcación del Segura, las medidas ambientales, excluidas las de saneamiento y depuración, son del 10,49\% del total. En la demarcación del Guadalquivir, sólo el $6 \%$ de las medidas están destinadas a la recuperación ambiental y no se prevén medidas para garantizar el establecimiento de los caudales ecológicos. En Cuencas Mediterráneas Andaluzas el presupuesto para recuperación ambiental es mínimo. De nuevo la baja proporción presupuestaria para medidas específicamente ambientales constituye un denominador común. Esta baja dotación presupuestaria para medidas ambientales básicas mientras se asigna una proporción predominante del presupuesto a medidas de satisfacción de las demandas y obras hidráulicas ajenas a los objetivos de la DMA, muestra que la alusión a una baja disponibilidad presupuestaria como justificación genérica para no establecer medidas ambientales no responde a la realidad.
Por otra parte, de forma generalizada se incluyen medidas implantadas antes de la adopción de los planes hidrológicos, cuando tales medidas debían haberse incluido en un análisis de referencias. Muchas de estas medidas son ajenas a la DMA y se centran fundamentalmente en infraestructuras hidráulicas, como ocurre en los casos del Ebro, el Segura, Cuencas Mediterráneas Andaluzas, Miño-Sil y Baleares. En la Demarcación del Ebro, el Programa de Medidas incluye infraestructuras hidráulicas ya aprobadas o cuya tramitación sigue adelante completamente al margen del proceso de planificación (embalse de Mularroya, embalse de Biscarrués, recrecimiento de Yesa, presa de Aguaviva).

Otro de los temas clave en relación con los Programas de Medidas es en qué grado reducen las presiones en la cuenca, por ejemplo a través del ahorro neto de agua y cambios en los patrones de cosechas o en los usos del suelo. En muchas demarcaciones obtener una reducción relevante de las presiones implica necesariamente aplicar medidas ambiciosas en el regadío, dado que en demarcaciones como el Guadalquivir, Guadiana, Segura, Ebro y otras, el regadío es uno de los problemas clave que impiden alcanzar los objetivos de buen estado. En estas demarcaciones el plan hidrológico es rehén del regadío, que puede representar el $80 \%$ o más de las demandas. Sin embargo, los planes no establecen medidas que aborden seriamente una reducción de la presión del regadío. De hecho, varios planes incluyen la creación de nuevos regadíos, particularmente en el caso del Ebro (158.000 ha. en el horizonte 2015 y otras 288.000 ha. entre 2015 y 2027) y del Guadalquivir (32.000 ha. en el horizonte 2015). Igualmente en la Demarcación del Guadiana ha tenido lugar una significativa transformación de cultivos leñosos de secano en regadío.

Para reducir los impactos del regadío, la medida estrella de la gran mayoría de planes hidrológicos son los planes de modernización de regadíos. Sin embargo, no están nada claros los resultados de estas millonarias inversiones en modernización, ni en términos de ahorro neto ni en términos de revisión de concesiones.

El argumento utilizado para apostar por la modernización de regadíos es el importante ahorro de agua que producirá. Pero esta hipótesis no está avalada con datos. En general, se contabilizan ahorros brutos del orden del $25-35 \%$ pero la disminución de la demanda neta se reduce a un $10-15 \%$, puesto que menguan mucho los retornos de riego a los cauces o infiltrados en los acuíferos. Según los planes de desarrollo rural, el ahorro neto se situaría en un $5-20 \%$ del ahorro potencial. De hecho, en Andalucía, uno de los pocos ámbitos con información sobre los resultados de los planes de modernización, los ahorros de agua de la modernización se han empleado en las 290.000 nuevas ha. de nuevos regadíos entre 1997 y 2008, de forma que no ha existido un ahorro real de recursos hídricos y las masas de las que se captan, en Andalucía mayoritariamente de aguas subterráneas, han mantenido 0 empeorado su mal estado cuantitativo.

También en la demarcación del Segura y otras los ahorros de la modernización previstos no suponen una menor detracción de agua, por lo que la consideración de la modernización como una medida ambiental (como ocurre en las demarcaciones del Guadalquivir o del Júcar) puede resultar un fraude. Los supuestos ahorros se destinan a aumentar la intensidad de la explotación 0 
a cultivar especies con mayores necesidades hídricas, aumentar la dotación de los llamados regadíos infradotados y, en alguna medida, aumentar la superficie efectiva del regadío.

Por otra parte, no se ha llevado a cabo la revisión de concesiones en casos clamorosos como concesiones en desuso o dotaciones incompatibles con el Plan. En algunos casos, como por ejemplo en el caso de la Comunidad de Regantes de Estremera en la Cuenca del Tajo, los caudales "ahorrados" se vendieron a los regantes de la cuenca del Segura durante la sequía 2005-2008, pervirtiendo así completamente el objetivo de una modernización subvencionada con fondos públicos.

Asimismo, no se están tomando medidas contundentes para controlar las extracciones ilegales, ni siquiera en espacios protegidos tan emblemáticos como Doñana, amenazado de forma directa por extracciones ilegales y donde no se tiene constancia del cierre de pozo alguno en la zona ni aparecen medidas de este tipo en el Plan del Guadalquivir. En el caso del Segura, se mantiene la perniciosa política de hechos consumados y amnistía a los regadíos ilegales de épocas pasadas, dado que su Normativa incluye la regularización de regadíos de hecho, consagrando la ampliación irregular del regadío como una vía ampliamente aceptada y aceptable para el organismo de cuenca. También en el Guadalquivir se han legalizado muchas captaciones ilegales y se han otorgado concesiones que llevaban años paradas o denegadas.

En relación con el cambio climático y los riesgos climáticos, existen comunidades autónomas con buenas prácticas en este ámbito, particularmente Cataluña, País Vasco, Andalucía y Navarra, pero en la generalidad de planes hidrológicos se observan serias carencias. Los planes hidrológicos se han limitado a aplicar los porcentajes de reducción contenidos en la Instrucción de Planificación Hidrológica para el cálculo de los recursos disponibles en 2027, sin que en la mayoría de los casos se ofrezcan datos actualizados acerca de los efectos del cambio climático sobre la disponibilidad de agua.

En relación con las infraestructuras verdes y la mitigación del riesgo de inundaciones, un patrón común en la generalidad de planes hidrológicos es que apenas existen medidas de gestión territorial, menos aún de traslado de infraestructuras u otras acciones para resolver los numerosos casos de ocupación del dominio público hidráulico y otras zonas inundables. Entre otros ejemplos, en el caso del Guadalquivir, el Plan no incorpora adecuadamente en su normativa las determinaciones de los instrumentos de Ordenación Territorial respecto a la red de drenaje.

Respecto a las medidas de restauración fluvial, hay que consignar algunas actuaciones importantes realizadas por las confederaciones hidrográficas del Duero y el Ebro y otras actuaciones dispersas pero interesantes en el Cantábrico, Tajo, cuencas internas de Cataluña, Guadiana y Guadalete-Barbate en Andalucía. Sin embargo, salvo media docena de actuaciones bandera, el resto son pequeñas obras o mejoras que no afectan, por ejemplo, a concesiones, explotación de embalses o demandas preexistentes. Por otra parte, en muchos programas de medidas aparecen actuaciones mal llamadas de restauración fluvial, que en absoluto podrían considerarse como tales.

\section{Programas de Seguimiento}

En relación con la operatividad de los programas de monitoreo y seguimiento, la situación general se caracteriza por serias carencias y en la actualidad por un parón generalizado ligado a limitaciones presupuestarias. En Baleares, desde 2010 no se ha hecho seguimiento de las aguas superficiales, ni epicontinentales, ni costeras. Igualmente los programas de seguimiento se han paralizado o no están plenamente operativos en otras muchas demarcaciones, como la del Guadiana o la del Ebro, incluyendo áreas protegidas como el Delta del Ebro.

Por otra parte, en la mayoría de demarcaciones la disponibilidad de datos se centra en pluviometría, caudales y estado de los embalses, mientras que faltan datos relativos a indicadores biológicos, fisicoquímicos e hidromorfológicos, que son claves para la DMA.

\section{AguAS SUBTERRÁNEAS}

Los planes hidrológicos ofrecen una visión aparentemente clara de los niveles de explotación de cada masa de agua subterránea, sin embargo ello contrasta con la escasa cantidad, calidad y nivel de actualización de los datos ofrecidos por los organismos de cuenca y su propio reconocimiento de la falta de registros de usos del agua y carencias en investigación básica en aguas subterráneas.

En relación con la metodología y datos disponibles para la clasificación del estado cuantitativo de las masas de agua subterránea, en muchas demarcaciones siguen existiendo serias lagunas de información, no sólo en relación con las extracciones, en las que existe una generalizada falta de control, con cientos de miles de pozos ilegales y escasa información contrastable sobre los volúmenes bombeados, sino también en relación con los recursos disponibles. En demarcaciones como la del Segura y otras, los datos disponibles no están actualizados, no reflejan las tendencias temporales, no están espacialmente analizados de forma adecuada y las metodologías aplicadas para los cálculos de balances y recursos no están suficientemente explicitadas 0 verificadas. Esta situación caracteriza también a otros muchos planes hidrológicos.

Por otra parte, como indicador de estado cuantitativo se ha utilizado de forma generalizada la relación entre extracciones/ recursos, de forma que no se han aplicado todos los criterios de clasificación del estado cuantitativo del Anexo V.2.1 de la DMA (véase por ejemplo el Plan del Guadiana). Sin embargo, la relación extracciones/recursos como indicador de estado cuantitativo presenta numerosas incertidumbres. Su valor tampoco refleja necesariamente la buena o mala calidad de los ecosistemas asociados. El estado cuantitativo debería medirse prioritariamente por la evolución en continuo de caudales surgentes y niveles piezométricos, cuyo uso está perfectamente en sintonía con la DMA, que prevé el control del estado cuantitativo a través de redes de seguimiento. A ello se añade que el mantenimiento de los manantiales representa una de las funciones ambientales más importan- 
tes de las aguas subterráneas, dando lugar al mantenimiento de ecosistemas ligados al agua y valores asociados de biodiversidad y paisaje. Por ello sorprende la escasa atención en la demarcación del Segura, la del Guadalquivir y otros muchos planes hidrológicos hacia la importancia del mantenimiento de fuentes y manantiales y sus funciones ambientales y sociales asociadas y sorprende su no inclusión como indicador clave del estado cuantitativo de las masas de agua subterránea.

Existen bastantes demarcaciones con serios problemas para alcanzar el buen estado de las masas subterráneas (casos como las demarcaciones del Tajo, Guadiana, Segura, Guadalate-Barbate, Tinto, Odiel y Piedras o Cuencas Internas de Cataluña), pese a lo cual las medidas previstas en estas masas son muy insuficientes. Como ejemplo, en el Segura no hay medidas específicas para mejorar el estado de las masas subterráneas pese a que el $73 \%$ no alcanzará el buen estado en 2015.

\section{EvALUACIÓN AMBIENTAL ESTRATÉGICA}

Los Informes de Sostenibilidad Ambiental (ISA) de la Evaluación Ambiental Estratégica (EAE) siguen de forma generalizada un mismo esquema (véase los casos de los planes hidrológicos del Ebro, Galicia-Costa, Miño-Sil, Cuencas Mediterráneas Andaluzas y otras), en el que destaca un análisis de alternativas carente de sentido ya que, con alguna variación, se contemplan dos o tres alternativas, una de las cuales es la alternativa ( 0 ) no hacer nada nuevo, a la que se añade una o dos alternativas más: (1) cumplir con las obligaciones establecidas en la Directiva en su forma más básica y (2) cumplir las obligaciones de la DMA en una forma más exigente. Por tanto, se plantea una alternativa ilegal y por tanto descartable y descartada previamente, que es la posibilidad de incumplir voluntariamente la Directiva Marco del Agua. Con respecto a las otras dos alternativas genéricas, la primera se limita a reproducir los contenidos del Plan y a enfrentarlos a una alternativa ambientalmente más ambiciosa, que queda descartada basada en genéricos argumentos de costes socioeconómicos. Por tanto, dichas alternativas no son tales, lo que desvirtúa los efectos reales de la EAE.

Frente a ello, los procedimientos de EAE no han analizado las alternativas concretas de gestión para los problemas clave de cada demarcación o los impactos del Plan y sus determinaciones sobre la Red Natura 2000. Por ejemplo, en la demarcación del Tajo no se identifica el trasvase Tajo-Segura como problema y, puesto que la identificación de los problemas ambientales ha sido la base para la propuesta de alternativas, ello supone que de facto no se hayan evaluado opciones distintas al trasvase, lo que invalida por completo el análisis de alternativas. En la Demarcación del Ebro el Informe de Sostenibilidad Ambiental renuncia expresamente al análisis de los efectos de cada una de las medidas propuestas, justificado sobre la supuesta complejidad de dicho análisis.

Urge una auditoría técnica y jurídica que analice la aplicación práctica de la EAE en España.

\section{LA APLICACIÓN DE LA DMA EN ALGUNOS CASOS EMBLEMÁticos}

La escasa -o nula - ambición a la hora de aplicar la Directiva y el mantenimiento de las viejas inercias de fomento de la obra hidráulica y ajenas a los objetivos ambientales de la DMA quedan magistralmente ejemplificados en casos tan relevantes como el Delta del Ebro, la Cuenca Alta del Guadiana, Doñana, l'Albufera de Valencia y el Estuario del Guadalquivir, como se describe sucintamente a continuación.

\section{Delta del Ebro}

En el caso del Delta del Ebro, ha habido falta de coordinación entre la CHE (Confederación Hidrográfica del Ebro) y el ACA (Agencia Catalana del Agua) en la designación y delimitación de las masas de agua del Delta del Ebro y del tramo final del río Ebro, así como en la adaptación de los índices de calidad ecológica para las aguas de transición.

En el caso de la determinación de caudales ambientales, la CHE ha prescindido de los datos y los resultados del ACA, y ha utilizado datos y argumentos poco contrastados. La planificación hidrológica ha obviado su estatus de zona protegida y no ha contemplado objetivos más exigentes ni medidas especiales para las masas de agua protegidas.

Por otra parte, se han declarado algunas masas de agua como fuertemente modificadas sin suficiente justificación, ya que de hecho es posible la consecución del buen estado ecológico con medidas técnicamente sencillas y económicamente viables.

Además, las medidas propuestas no se han realizado en su gran mayoría, a excepción de algunas de las que ya estaban programadas antes del 2009 en el Plan Integral de Protección del Delta del Ebro.

\section{Cuenca Alta del Guadiana}

El Plan Hidrológico del Guadiana explicita cuáles son los impactos que conforman la degradación de la Subcuenca Alta del Guadiana, con siete de las ocho masas de agua subterránea del Alto Guadiana en mal estado cuantitativo, seis de ellas en mal estado químico por contaminación con nitratos. Sin embargo, el Plan no adopta medidas consecuentes para reducir tales impactos, muy al contrario, va en la línea de consolidar de forma permanente los derechos de riego. En 2013 se aprueba el Plan y se elimina el Plan de Protección Especial del acuífero del Campo de Montiel, pese a que los derechos consolidados de uso de agua para regadíos duplican o triplican los recursos máximos disponibles, según datos oficiales de la CHG. Además, recientemente se han ampliado las dotaciones para el Campo de Montiel y en los últimos tres años se han incrementado un $34 \%$ los derechos consolidados.

La intensa sobreexplotación de las masas de agua subterránea está afectando a los espacios protegidos de la Subcuenca Alta del Guadiana, donde se hallan diversos LIC y ZEPA dependientes 
del agua, seis sitios Ramsar, la Reserva de la Biosfera de la Mancha Húmeda y un Parque Nacional.

Por otra parte, a pesar de las sentencias del Tribunal Supremo reconociendo la afección al Dominio Público de los Ojos del Guadiana y las Lagunas de Ruidera, no se han efectuado las tareas de deslinde y recuperación del mismo.

\section{Estuario del Guadalquivir}

En relación con el proyecto de la Autoridad Portuaria de Sevilla de dragado del tramo final del Guadalquivir, en 2010 la Comisión Científica establecida para valorar dicho proyecto recomendó la desestimación definitiva del mismo. El Borrador de Plan Hidrológico, presentado en 2010, no hacía referencia alguna al proyecto de profundización del dragado. Sin embargo, el Plan Hidrológico del Guadalquivir, aprobado en 2013, incluyó el dragado de profundización, aceptando las modificaciones y afecciones que ello supone para las masas implicadas. El Plan aprobado en 2013 hace así caso omiso a los conocimientos disponibles y a los pronunciamientos en contra de todas las instituciones, incluido el Patrimonio Mundial de UNESCO, el Consejo de Participación y la Comisión Científica específicamente creada para evaluar este proyecto.

Este proyecto ha supuesto que la Comisión Europea haya abierto un procedimiento de infracción contra España por el proyecto de dragado del Guadalquivir, pese a lo cual las autoridades pretenden continuar adelante con el mismo.

\section{Doñana}

El principal problema de gestión del agua en Doñana es actualmente la sobreexplotación del acuífero Almonte-Marismas (UH 05.51), compartido con la Demarcación del Tinto-Odiel-Piedras. Según el Plan Hidrológico de la Demarcación del Guadalquivir dicha masa está en buen estado cuantitativo, sin embargo, hay datos suficientes, incluyendo documentos oficiales y artículos científicos publicados, que sustentan de forma consistente, que dicha masa debe ser declarada en mal estado y por lo tanto "en riesgo" de incumplir la DMA. De hecho, tal conclusión es la que alcanza el Esquema de Temas Importantes (ETI) aprobado en 2010.

En relación con la información manejada en los datos de extracciones, la gran cantidad de pozos ilegales presentes en la zona indican que éstas podrían ser mayores que las estimadas. El mal estado del acuífero se evidencia en los niveles piezométricos, que indican claramente que la masa UH 05.51 ha mostrado tendencias descendentes continuas en su nivel freático en los últimos 40 años, a lo que se une la reducción de caudales que el acuífero aporta a los arroyos, lagunas y marismas.

Con respecto a las medidas, frente a los 30 millones de euros para el dragado del Guadalquivir (que el Plan incluye, irónicamente, como medida de "restauración morfológica"), no hay inversión nueva para Doñana.

Frente a esta realidad, las medidas que se requieren incluyen la constitución de comunidades de usuarios, la imposibilidad de otorgar nuevas concesiones y la elaboración de un plan de extracciones, como elementos imprescindibles para asegurar la recuperación de los niveles del acuífero y asegurar el futuro de Doñana.

\section{L'Albufera de Valencia}

La masa de agua tipo lago "l'Albufera de Valencia", incluida en el Parque Natural de l'Albufera de Valencia y declarada también LIC, ZEPA y área Ramsar, presenta un estado hipereutrófico por los retornos agrícolas y vertidos urbanos. El Plan Hidrológico del Júcar la ha clasificado como masa de agua muy modificada y retrasa el objetivo de alcanzar el buen potencial ecológico hasta el 2027. La excepción al logro de los objetivos ambientales en el horizonte 2015 para la l'Albufera de Valencia es incompatible con el artículo 4.1.c de la DMA, puesto que no permite el cumplimiento de las Directivas Hábitats y Aves, lo que redunda además, en el incumplimiento del artículo 4.2 de la DMA.

Además, la definición de "buen potencial ecológico" a alcanzar en 2027 no sólo es incompleta -pues no tiene en cuenta todos los indicadores de calidad biológica, hidromorfológica y físico-química requeridos por la DMA- sino que establece como objetivo en dicha fecha un valor medio de clorofila $30 \mu \mathrm{g} / \mathrm{l}$, lo que según los estudios científicos disponibles representa un objetivo de calidad ecológica entre aceptable y mala, mientras que para 2021 se asume el mal estado ecológico (valor medio de clorofila $90 \mu \mathrm{g} / \mathrm{l}$, estado hipereutrófico).

Por otra parte, los estudios científicos disponibles y el Informe para la Comisión Europea sobre la Conducción Júcar-Vinalopó, elaborado en 2004 por la Confederación Hidrográfica del Júcar, señalan que para alcanzar un estado aceptable, cercano al bueno, es necesario aportar un volumen anual de $253 \mathrm{hm}^{3}$ con una concentración media de fósforo no superior a $0,047 \mathrm{mg} / \mathrm{l}$, mientras que el actual Plan del Júcar fija un volumen de $167 \mathrm{hm}^{3}$ de requerimientos hídricos a garantizar, sin especificar la calidad mínima que deberían tener dichas aguas. 